Metadata for the data produced and analyzed for the article:

\title{
Filtration of Nordic recirculating aquaculture system wastewater: effects on microalgal growth, nutrient removal and nutritional value
}

Marco Calderini ${ }^{1 *}$, Čedomir Stevčić ${ }^{1}$, Sami Taipale $^{1}$, Katja Pulkkinen ${ }^{1}$

${ }^{1}$ Department of Biological and Environmental Science, Survontie 9C (YAC Building), Po Box 35, FI-40014, University of Jyväskylä, marco.92.calderini@jyu.fi. *Corresponding author.

The data processed and analyzed in this study is divided if five files: Growth_and_Nutrient_removal.txt, FA_concentration.txt, FA_proportion.txt, AA_concentration.txt and AA_proportion.txt. For detailed descriptions of the motives, methods and results of the study, see the article.

In the file Growth_and_Nutrient_removal.txt, the first column indicates the algal species studied: $\mathrm{HP}=$ Haematococcus pluvialis, MG = Monoraphidium griffithii, $\mathrm{SE}=$ Selenastrum sp.. Column 2 indicates the treatment, $W W=$ unfiltered RAS wastewater, FWW = filtered RAS wastewater. Column 3 represents the experimental run (1-2, for further details, see materials and methods in the article). Columns $4-7$ are density, specific growth rate, biomass and chlorophyll a (respectively) and they correspond to Figure 1 ad (respectively) of the article. Columns $8-11$ are percentage of nitrogen removed, percentage of phosphate removed, nitrogen removal rate and phosphate removal rate (respectively) and they correspond to Figure 2 a-d (respectively) of the article.

In the file FA_concentration.txt, the first column indicates the species: HP = Haematococcus pluvialis, MG $=$ Monoraphidium griffithii, $\mathrm{SE}=$ Selenastrum sp.. Column 2 indicates the treatment, WW $=$ unfiltered RAS wastewater, FWW = filtered RAS wastewater. Column 3 represents the experimental run (1-2, see materials and methods). Columns $4-21$ are the microalgal fatty acid concentrations identified through GC-MS that corresponded to more than $>0.5 \%$ of the total fatty acid concentration. The concentration of each fatty acid is given in $\mu \mathrm{g}$ of fatty acid per $\mathrm{mg}$ of microalgae dry weight. Sum of all $\omega-3$ and $\omega-6$ fatty acids are represented in the figures $3 \mathrm{~b}$ and $\mathrm{d}$ (respectively) of the article. Column 22 indicates the ratio between the sum of all $\omega-3$ and $\omega-6$ fatty acids. Column $23-27$ indicate actinobacterial fatty acids identified by GC-MS in microalgal samples. The value given to each of these fatty acids represents the concentration in $\mathrm{ng}$ of fatty acid per $\mathrm{mL}$ of filtered microalgae media. The sum of all actinobacterial fatty acid values were used to produce figure 6 of the article.

In the file FA_proportion.txt, the first column indicates the species: HP = Haematococcus pluvialis, MG = Monoraphidium griffithii, SE = Selenastrum sp.. Column 2 indicates the treatment, WW $=$ unfiltered RAS wastewater, FWW = filtered RAS wastewater. Columns $3-7$ represent the proportion of each $\omega-3$ fatty acid to the total $\omega-3$ fatty acids, calculated as: (concentration of $\omega-3$ fatty acid [I] ) / ( total concentration of $\omega-3$ fatty acids), with [I] representing any $\omega-3$ fatty acid.

Columns 8 - 12 represent the proportion of each $\omega-6$ fatty acid to the total $\omega-6$ fatty acids, calculated as: (concentration of $\omega-6$ fatty acid $[I]$ ) / (total concentration of $\omega-6$ fatty acids), with [I] representing any $\omega-6$ fatty acid. Proportion values of $\omega-3$ and $\omega-6$ fatty acids were used to perform PERMANOVA analysis. PERMANOVA results are represented in Table 2 of the articule, and results 
of Permutational Analysis of Multivariate Dispersion (PERMDISP) of the proportion data are presented in the Table 3 of the article.

In the file AA_concentration.txt, the first column indicates the species: HP = Haematococcus pluvialis, MG = Monoraphidium griffithii, $\mathrm{SE}=$ Selenastrum sp.. Column 2 indicates the treatment, WW $=$ unfiltered RAS wastewater, FWW = filtered RAS wastewater. Column 3 represents the experimental run (1-2, see materials and methods). Columns $4-18$ are the microalgal amino acid concentration identified through GC-MS. The concentration of each amino acid is given in $\mu \mathrm{g}$ of fatty acid per $\mathrm{mg}$ of microalgae dry weight. Columns 19 and 20 represent the sum of the concentration of all essential amino acids and non-essential amino acids, respectively. Mean microalgal essential and non-essential amino acids concentration values are represented in the Figure $4 \mathrm{~b}$ of the article.

In the file AA_proportion.txt, the first column indicates the species: $\mathrm{HP}=$ Haematococcus pluvialis, $\mathrm{MG}=$ Monoraphidium griffithii, SE = Selenastrum sp. Column 2 indicates the treatment, WW = unfiltered RAS wastewater, FWW = filtered RAS wastewater. Columns 3-10 represent the proportion of each essential amino acid to the total essential amino acids, calculated as: (concentration of essential amino acid [I]) / (total concentration of essential acids), with [I] representing any essential amino acid. Columns 11 - 17 represent the proportion of each non-essential amino acid to the total non-essential amino acids, calculated as: (concentration of non - essential amino acid [I]) / (total concentration of non - essential acids) , with $[I]$ representing any non-essential amino acid. Proportion values of essential and non-essential amino acids were used to perform PERMANOVA analysis. PERMANOVA results are represented in Table 2 of the articule, and results of Permutational Analysis of Multivariate Dispersion (PERMDISP) of the proportion data are presented in the Table 3 of the article. 